\title{
Public interest in rheumatic diseases and rheumatologist in the United States during the COVID-19 pandemic: evidence from Google Trends
}

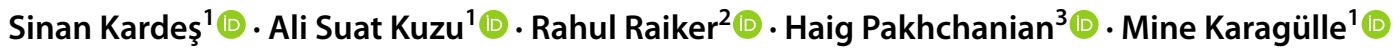

Received: 10 September 2020 / Accepted: 8 October 2020 / Published online: 18 October 2020

(c) Springer-Verlag GmbH Germany, part of Springer Nature 2020

\begin{abstract}
To evaluate the public interest in rheumatic diseases during the coronavirus disease 2019 (COVID-19) pandemic. Google Trends was queried to analyze search trends in the United States for numerous rheumatic diseases and also the interest in a rheumatologist. Three 8-week periods in 2020 ((March 15-May 9), (May 10-July 4), and (July 5-August 29)) were compared to similar periods of the prior 4 years (2016-2019). Compared to a similar time period between 2016 and 2019, a significant decrease was found in the relative search volume for more than half of the search terms during the initial March 15-May 9, 2020 period. However, this trend appeared to reverse during the July 5-August 29, 2020 period where the relative volume for nearly half of the search terms were not statistically significant compared to similar periods of the prior 4 years. In addition, this period showed a significant increase in relative volume for the terms: Axial spondyloarthritis, ankylosing spondylitis, psoriatic arthritis, rheumatoid arthritis, Sjögren's syndrome, antiphospholipid syndrome, scleroderma, Kawasaki disease, Anti-Neutrophil Cytoplasmic Antibody (ANCA)-associated vasculitis, and rheumatologist. There was a significant decrease in relative search volume for many rheumatic diseases between March 15 and May 9, 2020 when compared to similar periods during the prior 4 years. However, the trends reversed after the initial period ended. There was an increase in relative search for the term "rheumatologist" between July and August 2020 suggesting the need for rheumatologists during the COVID19 pandemic. Policymakers and healthcare providers should address the informational demands on rheumatic diseases and needs for rheumatologists by the general public during pandemics like COVID-19.
\end{abstract}

Keywords Google search $\cdot$ Internet $\cdot$ Rheumatic disease $\cdot$ Rheumatologist

Sinan Kardeş

sinan.kardes@istanbul.edu.tr

Ali Suat Kuzu

alisuatkuzu@gmail.com

Rahul Raiker

rsr0010@mix.wvu.edu

Haig Pakhchanian

haig@gwmail.gwu.edu

Mine Karagülle

mkgulle@istanbul.edu.tr

1 Department of Medical Ecology and Hydroclimatology, Istanbul Faculty of Medicine, Istanbul University, Capa-Fatih, 34093 Istanbul, Turkey

2 West Virginia University School of Medicine, Morgantown, WV, USA

3 George Washington University School of Medicine \& Health Science, Washington, DC, USA

\section{Introduction}

The recent pandemic of coronavirus disease 2019 (COVID19) has placed the rheumatologists and immunologists at the forefront of understanding the pathogenesis and management of this infection [1-4]. After severe acute respiratory syndrome coronavirus 2 (SARS-CoV-2) cellular infection, the host immune response is triggered and generates proinflammatory cytokines and chemokines [5]. These molecules recruit monocytes, macrophages, and $\mathrm{T}$ cells which leads to create further inflammation that inevitably establishes a pro-inflammatory feedback loop [5]. A defective immune response aggressively generates pro-inflammatory cytokines, called cytokine storms, which can lead to multiple-organ dysfunction [5]. Rheumatologists and immunologists have substantial expertise in the pathogenesis of the immune response, the impact of inflammation, and their treatments [1-4]. Several immunomodulatory and antiinflammatory drugs (e.g., hydroxychloroquine, colchicine, 
tocilizumab, anakinra) have been used for COVID-19 when scientists are in search of a possible treatment for this novel virus and a resultant inflammatory response [1-4, 6-8]. Since rheumatologists have substantial knowledge in the benefits and adverse effects of these drugs as well, rheumatologists are playing a significant role in this pandemic. Besides fighting COVID-19, the rheumatologists also have an essential role in caring for their rheumatic patients who may express concerns about their potential increased risk of acquiring COVID-19 infection due to their underlying disease itself and any immunosuppressive treatments they may be on [9-16].

Google Trends has been demonstrated as a powerful tool in surveying outbreaks and monitoring public interest [17-20]. It was used in previous outbreaks including influenza [21-23] and Zika virus [24]. Interestingly, for COVID19 pandemic, Google Trends was used to evaluate public interest in other medical disciplines including dermatology [25], plastic surgery [26], urology [27] and otolaryngology [28]. These studies highlight the integration of Google Trends to the field of medicine as a valuable epidemiologic data to monitor public interest.

The public interest in rheumatic diseases and rheumatologist during the pandemic represents a knowledge gap. This information may be useful to determine whether the public has access to rheumatological-related information that may guide to policymakers and healthcare providers to address the informational demands and needs for rheumatologists during the COVID-19 pandemic by the general public. Therefore, the aim of this study was to use the Google Trends data to evaluate the public interest in rheumatic diseases and rheumatologist during the COVID-19 pandemic.

\section{Methods}

Google Trends provides the relative interest of Google searches, on a range of 0-100 [29]. Larger scores indicate greater relative interest. A wide range of search terms were determined to represent nearly all rheumatic diseases that patients might search on Google (i.e., Osteoarthritis, gout, pseudogout, calcium pyrophosphate crystal deposition (CPPD), fibromyalgia, axial spondyloarthritis, ankylosing spondylitis, peripheral spondyloarthritis, psoriatic arthritis, reactive arthritis, septic arthritis, rheumatoid arthritis, Sjögren's syndrome, systemic lupus erythematosus, antiphospholipid syndrome, scleroderma, polymyositis, dermatomyositis, relapsing polychondritis, familial Mediterranean fever, Tumor Necrosis Factor (TNF) Receptor-Associated Periodic Syndrome (TRAPS), Hyperimmunoglobulinemia D with Periodic Fever Syndrome (HIDS), Cryopyrin-Associated Periodic Syndromes (CAPS), vasculitis, Takayasu arteritis, giant cell arteritis, temporal arteritis, polyarteritis nodosa, Kawasaki disease, polymyalgia rheumatica, Anti-Neutrophil Cytoplasmic Antibody (ANCA)associated vasculitis, granulomatosis with polyangiitis, and Behçet's syndrome). In addition, the term "rheumatologist" was also analyzed. Although some terms are used interchangeably for the disease (e.g., pseudogout and CPPD), the study included all listed terms because the general public may not search for synonymous terms. Each term was searched as presented except for calcium pyrophosphate crystal deposition (searched as CPPD), Sjögren's syndrome (as Sjogren), Systemic lupus erythematosus (as lupus), ANCA-associated vasculitis (as ANCA vasculitis), Behçet's syndrome (searched as Behcet). TRAPS and HIDS could not be included because Google Trends did not provide data for these two diseases due to their low relative search volume. On September 6, 2020, these search terms were queried on Google Trends using filters of "United States", "01/01/2016-09/06/2020", and "All categories". The Google Trends weekly data were downloaded.

Three 8-week periods in 2020 ((March 15-May 9), (May 10-July 4) and (July 5-August 29)) were compared to similar periods of the prior 4 years (2016-2019) to evaluate both initial- and short-term interest. Dates after March 15, 2020, when nationwide quarantine measures became implemented, were evaluated. Generalized estimating equations with gamma distribution were used in comparisons. Analyses were performed with SPSS 21.0. $P$ values less than 0.05 were considered statistically significant.

\section{Results}

In the March 15-May 9, 2020 period, the relative search volume for 21 of the 32 search terms (i.e., osteoarthritis, gout, pseudogout, CPPD, fibromyalgia, peripheral spondyloarthritis, reactive arthritis, septic arthritis, Sjögren's syndrome, scleroderma, polymyositis, dermatomyositis, CAPS, vasculitis, Takayasu arteritis, giant cell arteritis, temporal arteritis, polyarteritis nodosa, polymyalgia rheumatica, Behçet's syndrome, and rheumatologist) statistically significantly decreased; however, the search term (i.e., psoriatic arthritis) statistically significantly increased compared to prior 4 years (Table 1 ).

In the May 10-July 4, 2020 period, the relative search volume for 11 of the 32 search terms (i.e., osteoarthritis, pseudogout, fibromyalgia, peripheral spondyloarthritis, systemic lupus erythematosus, scleroderma, polymyositis, CAPS, Takayasu arteritis, temporal arteritis, and Behçet's syndrome) statistically significantly decreased; however, four search terms (i.e. axial spondyloarthritis, ankylosing spondylitis, psoriatic arthritis, and giant cell arteritis) statistically significantly increased compared to prior 4 years (Table 1). 


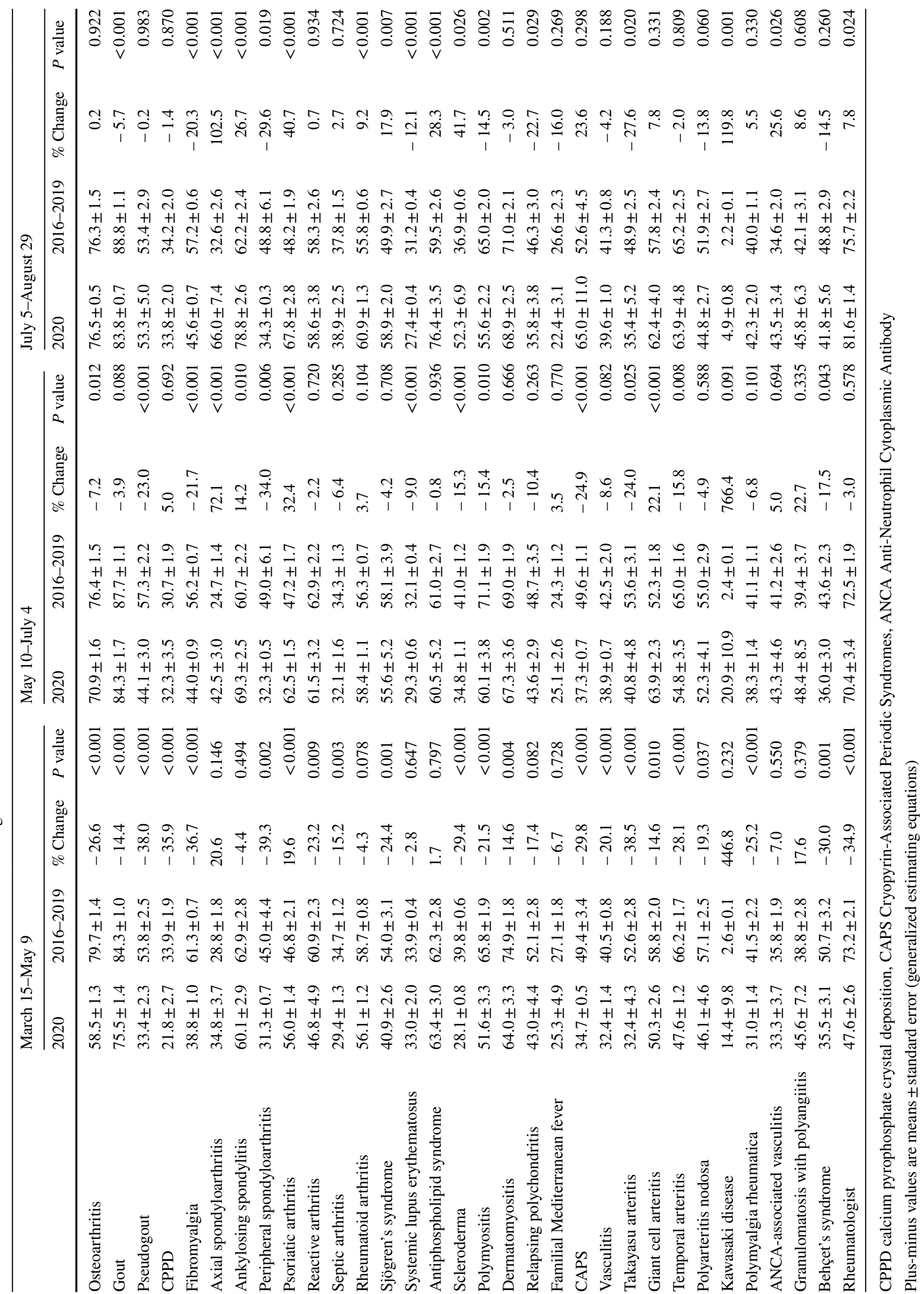


In the July 5-August 29, 2020 period, relative search volume of 7 of the 32 search terms (i.e., gout, fibromyalgia, peripheral spondyloarthritis, systemic lupus erythematosus, polymyositis, relapsing polychondritis, and Takayasu arteritis) statistically significantly decreased; however, 10 search terms (i.e., axial spondyloarthritis, ankylosing spondylitis, psoriatic arthritis, rheumatoid arthritis, Sjögren's syndrome, antiphospholipid syndrome, scleroderma, Kawasaki disease, ANCA-associated vasculitis, and rheumatologist) statistically significantly increased compared to prior 4 years (Table 1).

\section{Discussion}

The study found a statistically significant decrease in relative search volume of more than half of the search terms in the March 15-May 9, 2020 period. However, this trend appeared to reverse after this initial period. In the July 5-August 29, 2020 period, the relative volume of nearly half of the search terms were not significantly different when compared to similar periods of the prior 4 years; however, the terms axial spondyloarthritis, ankylosing spondylitis, psoriatic arthritis, rheumatoid arthritis, Sjögren's syndrome, antiphospholipid syndrome, scleroderma, Kawasaki disease, ANCA-associated vasculitis, and rheumatologist all statistically significantly increased compared to 4 years prior.

Previous studies evaluated the public interest in other medical disciplines during the COVID-19 pandemic [25-28]. Even though direct comparison of the present study is limited by differences in study design, the medical discipline evaluated, and the time periods compared, the present findings are consistent with those of the previous studies [25-28]. Each study has shown a significant decrease in relative search volume for most of the search terms in the initial COVID-19 pandemic period. Shifting interest of patients from their rheumatic disease to COVID-19 might be responsible for this finding in the initial period.

In the July 5-August 29, 2020 period, the relative search volume for many of the search terms were either not statistically significant or significantly increased compared to similar periods of the prior 4 years. This finding may indicate that the interest in rheumatic diseases has gradually recovered shortly after the initial COVID-19 period, and the general public has informational needs on a wide range of rheumatic diseases. Interestingly, psoriatic arthritis was increased in all three periods evaluated in the study with an increase in relative search volume of axial spondyloarthritis, ankylosing spondylitis, rheumatoid arthritis, Sjögren's syndrome, antiphospholipid syndrome, scleroderma, Kawasaki disease, and ANCA-associated vasculitis in the July/August period. The gradual increased interest in rheumatic diseases might be due to the general public's lack of knowledge on potential risk that may exist between these diseases and immunosuppressive treatments with COVID-19 [30, 31].

In a previous study using Google Trends, Dey and Zhao compared the relative search volume of Kawasaki disease during February-May 2020 to similar period in 2019 [32]. They showed a similar level of public interest in Kawasaki disease in February-March 2020 and 2019, and an increased interest after mid-April, 2020 [32]. The present study showed a similar increase in relative search of Kawasaki disease in all three periods evaluated while reaching a statistical significance during the July 5-August 29, 2020 period. The findings from the previous study (i.e., increasing interest after mid-April) [32] suggest that the insignificant increase between March 15 and May 9, 2020 may be due to similar relative search volume between March and early April, 2020. On the other hand, this study also found an increased interest in relative search of antiphospholipid syndrome. News on Kawasaki-like disease, and discussions on possible presence of antiphospholipid antibodies in COVID-19 might contribute to the observed trends in the present study [33-36].

It is interesting to note that there was no significant difference in relative search volume of the term "rheumatologist" between May 10 and July 4, 2020 compared to similar periods in 2016-2019; however, a statistically significant increase was seen in the July 5-August 29, 2020 period. This finding that indicates the increased need for rheumatologists during the COVID-19 pandemic may be attributable to two factors: (a) patients with a rheumatic disease and/or receiving immunosuppressive treatments wanted to consult rheumatologists on their disease/treatments [30]; (b) patients consult rheumatologists on properties of several anti-inflammatory and immunomodulatory drugs that are being used in the treatment of COVID-19 because rheumatologists are experts in the use of these drugs [1, 7]. Rheumatic patients may be at a higher risk of receiving COVID infection with worse outcomes, as many of them tend to be susceptible from their underlying rheumatic disease or immunocompromised due to their treatment [9-16]. Thus, this pandemic has put great responsibility on the shoulders of the rheumatologists for caring for this vulnerable patient group. In addition, considerable knowledge of rheumatologists and immunologists on the management of patients with pathological immune responses coupled with their substantial clinical experiences with many of the drugs that are currently being tested as potential COVID-19 treatment has led to rheumatologists and immunologists being placed right in the frontlines in facing COVID-19 [1-4, 6-8]. All these demonstrate that the rheumatologists and immunologists have an indispensable role during the COVID-19 pandemic.

A limitation of this study is that the Google search engine was the only search engine used to conduct the analysis. However, because over $85 \%$ of internet users in the United States rely on Google for information searches [37], the data 
used for this study represent a large sample size of American search queries as a whole. Moreover, the study evaluated the public interest only in initial and short term; therefore, a study examining the long-term interest during the pandemic is warranted in the future. In addition, the trends in COVID19 terms, which would allow a better interpretation of the present results, could not be included to avoid overlapping publication with previous studies examining the trends of public interest in these terms $[38,39]$. Another limitation of this study is that it does not provide information regarding the reasons for the observed trends. Further research is necessary to address this important issue.

In conclusion, there was a significant decrease in relative search volume for many rheumatic diseases between March 15 and May 9, 2020 when compared to similar periods during the prior 4 years. However, the trends reversed after the initial period ended. There was an increase in relative search for the term "rheumatologist" between July and August 2020 suggesting the need for rheumatologists during the COVID19 pandemic. Policymakers and healthcare providers should address the informational demands on rheumatic diseases and needs for rheumatologists by the general public during pandemics like COVID-19.

\section{Acknowledgements None.}

Author contributions Conception or design of the study: SK; data collection, analysis, and/or interpretation: SK, ASK, RR, HP, and MK; drafting the article: SK; critical revision of the article: SK, ASK, RR, $\mathrm{HP}$, and MK; final approval of the version of the article to be published: SK, ASK, RR, HP, and MK

\section{Funding None.}

Availability of data and materials Available from the corresponding author upon reasonable request.

\section{Compliance with ethical standards}

Conflict of interest The authors have no conflict of interest in this study.

Ethics approval Not applicable.

Consent to participate Not applicable.

Consent for publication Not applicable

\section{References}

1. Misra DP, Agarwal V, Gasparyan AY, Zimba O (2020) Rheumatologists' perspective on coronavirus disease 19 (COVID-19) and potential therapeutic targets. Clin Rheumatol 39:2055-2062. https ://doi.org/10.1007/s10067-020-05073-9

2. Gheita TA, Salem MN, Eesa NN, Khalil NM, Gamal NM, Noor RA et al (2020) Rheumatologists' practice during the
Coronavirus disease 2019 (COVID-19) pandemic: a survey in Egypt. Rheumatol Int 40:1599-1611. https://doi.org/10.1007/ s00296-020-04655-9

3. Ladani AP, Loganathan M, Danve A (2020) Managing rheumatic diseases during COVID-19. Clin Rheumatol. https://doi. org/10.1007/s10067-020-05387-8

4. Batu ED, Özen S (2020) Implications of COVID-19 in pediatric rheumatology. Rheumatol Int 40:1193-1213. https://doi. org/10.1007/s00296-020-04612-6

5. Tay MZ, Poh CM, Rénia L, MacAry PA, Ng LFP (2020) The trinity of COVID-19: immunity, inflammation and intervention. Nat Rev Immunol 20:363-374. https://doi.org/10.1038/s4157 7-020-0311-8

6. Shah S, Das S, Jain A, Misra DP, Negi VS (2020) A systematic review of the prophylactic role of chloroquine and hydroxychloroquine in coronavirus disease-19 (COVID-19). Int J Rheum Dis 23:613-619. https://doi.org/10.1111/1756-185X.13842

7. Misra DP, Gasparyan AY, Zimba O (2020) Benefits and adverse effects of hydroxychloroquine, methotrexate and colchicine: searching for repurposable drug candidates. Rheumatol Int. https://doi.org/10.1007/s00296-020-04694-2

8. Gasparyan AY, Misra DP, Yessirkepov M, Zimba O (2020) Perspectives of Immune Therapy in Coronavirus Disease 2019. J Korean Med Sci 35:e176. https://doi.org/10.3346/jkms.2020.35. e176

9. Furer V, Rondaan C, Heijstek M et al (2019) Incidence and prevalence of vaccine preventable infections in adult patients with autoimmune inflammatory rheumatic diseases (AIIRD): a systemic literature review informing the 2019 update of the EULAR recommendations for vaccination in adult patients with AIIRD. RMD Open 5:e001041. https://doi.org/10.1136/rmdop en-2019-001041

10. Tam LS, Tanaka Y, Handa R et al (2020) Care for patients with rheumatic diseases during COVID-19 pandemic: A position statement from APLAR. Int J Rheum Dis 23:717-722. https://doi. org/10.1111/1756-185X.13863

11. Freites Nuñez DD, Leon L, Mucientes A et al (2020) Risk factors for hospital admissions related to COVID-19 in patients with autoimmune inflammatory rheumatic diseases. Ann Rheum Dis. https://doi.org/10.1136/annrheumdis-2020-217984

12. Santos CS, Morales CM, Álvarez ED, Castro CÁ, Robles AL, Sandoval TP (2020) Determinants of COVID-19 disease severity in patients with underlying rheumatic disease. Clin Rheumatol 39:2789-2796. https://doi.org/10.1007/s10067-020-05301-2

13. Koker O, Demirkan FG, Kayaalp G, Cakmak F, Tanatar A, Karadag SG, Sonmez HE, Omeroglu R, Aktay Ayaz N (2020) Does immunosuppressive treatment entail an additional risk for children with rheumatic diseases? A survey-based study in the era of COVID-19. Rheumatol Int 40:1613-1623. https://doi. org/10.1007/s00296-020-04663-9

14. Kastritis E, Kitas GD, Vassilopoulos D, Giannopoulos G, Dimopoulos MA, Sfikakis PP (2020) Systemic autoimmune diseases, anti-rheumatic therapies, COVID-19 infection risk and patient outcomes. Rheumatol Int 40:1353-1360. https://doi.org/10.1007/ s00296-020-04629-x

15. Haslak F, Yildiz M, Adrovic A, Sahin S, Koker O, Aliyeva A, Barut K, Kasapcopur O (2020) Management of childhood-onset autoinflammatory diseases during the COVID-19 pandemic. Rheumatol Int 40:1423-1431. https://doi.org/10.1007/s0029 6-020-04645-x

16. Montero F, Martínez-Barrio J, Serrano-Benavente B, González T, Rivera J, Molina Collada J, Castrejón I, Álvaro-Gracia J (2020) Coronavirus disease 2019 (COVID-19) in autoimmune and inflammatory conditions: clinical characteristics of poor outcomes. Rheumatol Int 40:1593-1598. https://doi.org/10.1007/ s00296-020-04676-4 
17. Eysenbach G (2009) Infodemiology and infoveillance: framework for an emerging set of public health informatics methods to analyze search, communication and publication behavior on the Internet. J Med Internet Res 11:e11. https://doi.org/10.2196/ jmir.1157

18. Mavragani A, Ochoa G (2019) Google Trends in infodemiology and infoveillance: methodology framework. JMIR Public Health Surveill 5:e13439. https://doi.org/10.2196/13439

19. Kardeş $S$ (2019a) Seasonal variation in the internet searches for gout: an ecological study. Clin Rheumatol 38:769-775. https:// doi.org/10.1007/s10067-018-4345-2

20. Kardeş S (2019b) Seasonal variation in the internet searches for psoriasis. Arch Dermatol Res 311:461-467. https://doi. org/10.1007/s00403-019-01921-0

21. Carneiro HA, Mylonakis E (2009) Google trends: a web-based tool for real-time surveillance of disease outbreaks. Clin Infect Dis 49:1557-1564. https://doi.org/10.1086/630200

22. Lu FS, Hou S, Baltrusaitis K et al (2018) Accurate influenza monitoring and forecasting using novel internet data streams: a case study in the Boston metropolis. JMIR Public Health Surveill 4:e4. https://doi.org/10.2196/publichealth.8950

23. Lampos V, Miller AC, Crossan S, Stefansen C (2015) Advances in nowcasting influenza-like illness rates using search query logs. Sci Rep 5:12760. https://doi.org/10.1038/srep12760

24. Teng Y, Bi D, Xie G et al (2017) Dynamic forecasting of Zika epidemics using Google Trends. PLoS ONE 12:e0165085. https ://doi.org/10.1371/journal.pone.0165085

25. Guzman AK, Barbieri JS (2020) Analysis of dermatology-related search engine trends during the COVID-19 pandemic: Implications for patient demand for outpatient services and telehealth. J Am Acad Dermatol 83:963-965. https://doi.org/10.1016/j. jaad.2020.05.147

26. Dhanda AK, Leverant E, Leshchuk K, Paskhover B (2020) A Google Trends analysis of facial plastic surgery interest during the COVID-19 pandemic. Aesthetic Plast Surg 44:1378-1380. https://doi.org/10.1007/s00266-020-01903-y

27. Bhambhvani HP, Tijerina JD, Parham MJ, Greenberg DR, Eisenberg ML (2020) Public interest in elective urologic procedures in the COVID-19 pandemic: A Google Trends analysis. Urol Pract. https://doi.org/10.1097/UPJ.0000000000000179

28. Pier MM, Pasick LJ, Benito DA, Alnouri G, Sataloff RT (2020) Otolaryngology-related Google Search trends during the COVID-19 pandemic. Am J Otolaryngol 41:102615. https://doi. org/10.1016/j.amjoto.2020.102615

29. Google Trends. https://trends.google.com/trends/. September 6, 2020
30. Seyahi E, Poyraz BC, Sut N, Akdogan S, Hamuryudan V (2020) The psychological state and changes in the routine of the patients with rheumatic diseases during the coronavirus disease (COVID19) outbreak in Turkey: a web-based cross-sectional survey. Rheumatol Int 40:1229-1238. https://doi.org/10.1007/s00296-02004626-0

31. Coskun Benlidayi I, Kurtaran B, Tirasci E, Guzel R (2020) Coronavirus disease 2019 (COVID-19) in a patient with ankylosing spondylitis treated with secukinumab: a case-based review. Rheumatol Int 40:1707-1716. https://doi.org/10.1007/s00296-02004635-z

32. Dey M, Zhao SS (2020) COVID-19 and Kawasaki disease: an analysis using Google Trends. Clin Rheumatol 39:2483-2484. https://doi.org/10.1007/s10067-020-05231-z

33. https://www.nytimes.com/2020/05/13/health/coronavirus-child ren-kawasaki-pmis.html/. Accessed September 9, 2020

34. Verdoni L, Mazza A, Gervasoni A et al (2020) An outbreak of severe Kawasaki-like disease at the Italian epicentre of the SARSCoV-2 epidemic: an observational cohort study. Lancet 395:17711778. https://doi.org/10.1016/S0140-6736(20)31103-X

35. Amezcua-Guerra LM, Rojas-Velasco G, Brianza-Padilla M et al (2020) Presence of antiphospholipid antibodies in COVID-19: case series study. Ann Rheum Dis. https://doi.org/10.1136/annrh eumdis-2020-218100

36. Ahmed S, Zimba O, Gasparyan AY (2020) Thrombosis in Coronavirus disease 2019 (COVID-19) through the prism of Virchow's triad. Clin Rheumatol 39:2529-2543. https://doi.org/10.1007/ s10067-020-05275-1

37. Statcounter. https://gs.statcounter.com/search-engine-marke t-share/. Accessed September 6, 2020

38. Husain I, Briggs B, Lefebvre C, Cline DM, Stopyra JP, O'Brien MC et al (2020) Fluctuation of public interest in COVID-19 in the United States: retrospective analysis of google trends search data. JMIR Public Health Surveill 6:e19969. https://doi. org/10.2196/19969

39. Mavragani A, Konstantinos G (2020) On the predictability of COVID-19 in USA: A Google Trends analysis. https://www. researchsquare.com/article/rs-27189/v1/. https://doi.org/10.21203 /rs.3.rs-27189/v1. (Preprint)

Publisher's Note Springer Nature remains neutral with regard to jurisdictional claims in published maps and institutional affiliations. 